\title{
Leveraging Cloud Computing to Address Public Health Disparities: An Analysis of the SPHPS
}

\author{
Arash Jalali, ${ }^{1,3,4}$ Olusegun A. Olabode, ${ }^{1,2}$ and Christopher M. Bell ${ }^{3,4}$ \\ ${ }^{1}$ University of Illinois at Chicago, School of Public Health \\ ${ }^{2}$ U. S. Department of Veterans Affairs and the Department of Defense (DoD), \\ Captain James A. Lovell Federal Health Care Center \\ ${ }^{3}$ University of Illinois Hospital \& Health Sciences System \\ ${ }^{4}$ University of Illinois at Chicago, Biomedical and Health Information Sciences
}

\section{Abstract}

As the use of certified electronic health record technology (CEHRT) has continued to gain prominence in hospitals and physician practices, public health agencies and health professionals have the ability to access health data through health information exchanges (HIE). With such knowledge health providers are well positioned to positively affect population health, and enhance health status or quality-of-life outcomes in at-risk populations. Through big data analytics, predictive analytics and cloud computing, public health agencies have the opportunity to observe emerging public health threats in real-time and provide more effective interventions addressing health disparities in our communities. The Smarter Public Health Prevention System (SPHPS) provides real-time reporting of potential public health threats to public health leaders through the use of a simple and efficient dashboard and links people with needed personal health services through mobile platforms for smartphones and tablets to promote and encourage healthy behaviors in our communities. The purpose of this working paper is to evaluate how a secure virtual private cloud (VPC) solution could facilitate the implementation of the SPHPS in order to address public health disparities.

Keywords: public health informatics, smarter public health prevention system, cloud computing, virtual private cloud, big data analytics, health disparities

\section{Background}

With the emergence of big data analytics, predictive analytics, and cloud computing, an information technology revolution is occurring in the field of public health informatics (PHI). PHI "is the science of applying Information-Age technology to serve the specialized needs of public health," 1 and is "the systematic application of information and computer science and technology to public health practice, research, and learning." ${ }^{2}$ PHI utilizes an interdisciplinary approach and methods from various disciplines, including: information science; computer science; management; organizational theory; psychology; communications; political science; law; and public health fields, to make information-driven decisions and present new-

knowledge. ${ }^{1,3,4}$ A comprehensive public health program should include a "broad social enterprise 
that seeks to extend the benefits of current knowledge in ways that will have the maximum impact on the health status of a population." ${ }^{33}$ A comprehensive public health program should also include strategic agility. Strategic agility is "the ability to capitalize on opportunities and dodge threats with speed and assurance." 5

Most cloud architectures are defined as "public" or "private." A VPC is neither and has the characteristics of both:

- "Public" clouds are open to the general Internet and are available to the public. These clouds are suited for services designed for wide usage by many people, and security in these clouds is usually dependent on the application and web servers installed in the cloud space, vs. the cloud space itself. In contrast, a VPC places security walls around the cloud space, restricting access to specific users, roles or machines, separate and predicate to the servers in the cloud address space.

- "Private" clouds are not open to the general Internet, and are usually available only to private users within the firewalls of an organization. Generally they refer to infrastructure already internal to an organization, which is organized and managed as a cloud. This is done to gain efficiencies similar to engaging external cloud vendors, yet keep control and security with familiar boundaries. In contrast, a VPC is cloud space outside of the firewall of an organization, but is often directly connected to internal networks via network connections.

The field of PHI is also affected by external rules and regulations changes occurring at the federal level. ${ }^{6}$ The Health Information Technology for Economic and Clinical Health Act (HITECH Act) was enacted as part of the of the American Recovery and Reinvestment Act of 2009 (ARRA) to promote health information technology (HIT) including health information exchanges (HIEs) and support the electronic use and exchange of health information, in addition to improving health care quality, safety, and efficiency. ${ }^{7}$ ARRA establishes HIT adoption as a national priority from patient-centric care to population wide health initiatives, which has the potential to improve population and public health outcomes. ${ }^{8,9}$

It is the goal of ARRA to promote electronic health record (EHR) adoption through Meaningful Use of CEHRT which fundamentally transforms the way public health professionals "monitor health threats and respond to injury, disease, and disability among the population." " One of the key concepts of EHR Meaningful Use is to "improve the health of the population" and public health. ${ }^{10}$ According to the National Priorities Partnership's National Priorities and Goals: Population Health ${ }^{10}$ "envision communities that foster health and wellness as well as national, state, and local systems of care fully invested in the prevention of disease, injury, and disability - reliable, effective, and proactive in helping all people reduce the risk and burden of disease." Additionally, the mission of National Prevention Council is to "increase the number of Americans who are healthy at every stage of life."11 The National Prevention Strategy will improve American health through four pillars which are "create healthy and safe communities, expand clinical and community-based preventive services, empower people to make healthy choices, and eliminate health disparities." 11 
The field of PHI is also affected by rapid speed of change of the globalized social enterprise and increasing technological innovations. ${ }^{5}$ Internet searches like Google's Flu Trends detect outbreaks of influenza in accelerated fashion. ${ }^{12,}{ }^{13}$ Conventional public health influenza surveillance systems are lacking behind Google Flu Trends. ${ }^{12,}{ }^{13}$ Without the adoption of cloud computing, public health programs cannot aggregate the data they need in other to get an actual picture of population health.

\section{Methodology}

Public health professionals will need to align public health information technology (PHIT) with national polices including the National Prevention Council's National Priorities Strategy. This alignment requires a more agile hardware and software computing infrastructure to support "stronger partnerships and coordination of care between the public health and health care delivery systems.", 10,11 A cloud computing infrastructure will help public health professionals to access real-time or near real-time data to respond to emerging public health threats including improving population health and elimination of health disparities in our communities. , $^{, 10,11}$

\section{What is a Virtual Private Cloud (VPC)?}

According to McDonald and Kapashi, VPC is a cloud space dedicated to a single project/entity. It usually implies that everything from "network-in" is unique to a specific entity or "tenant." This means that many resources associated with cloud deployments are defined, dedicated and are not shared with other cloud consumers/tenants, including:

- Virtual machines

- Databases

- Application Servers
- Repositories

- Directories

- Network address spaces

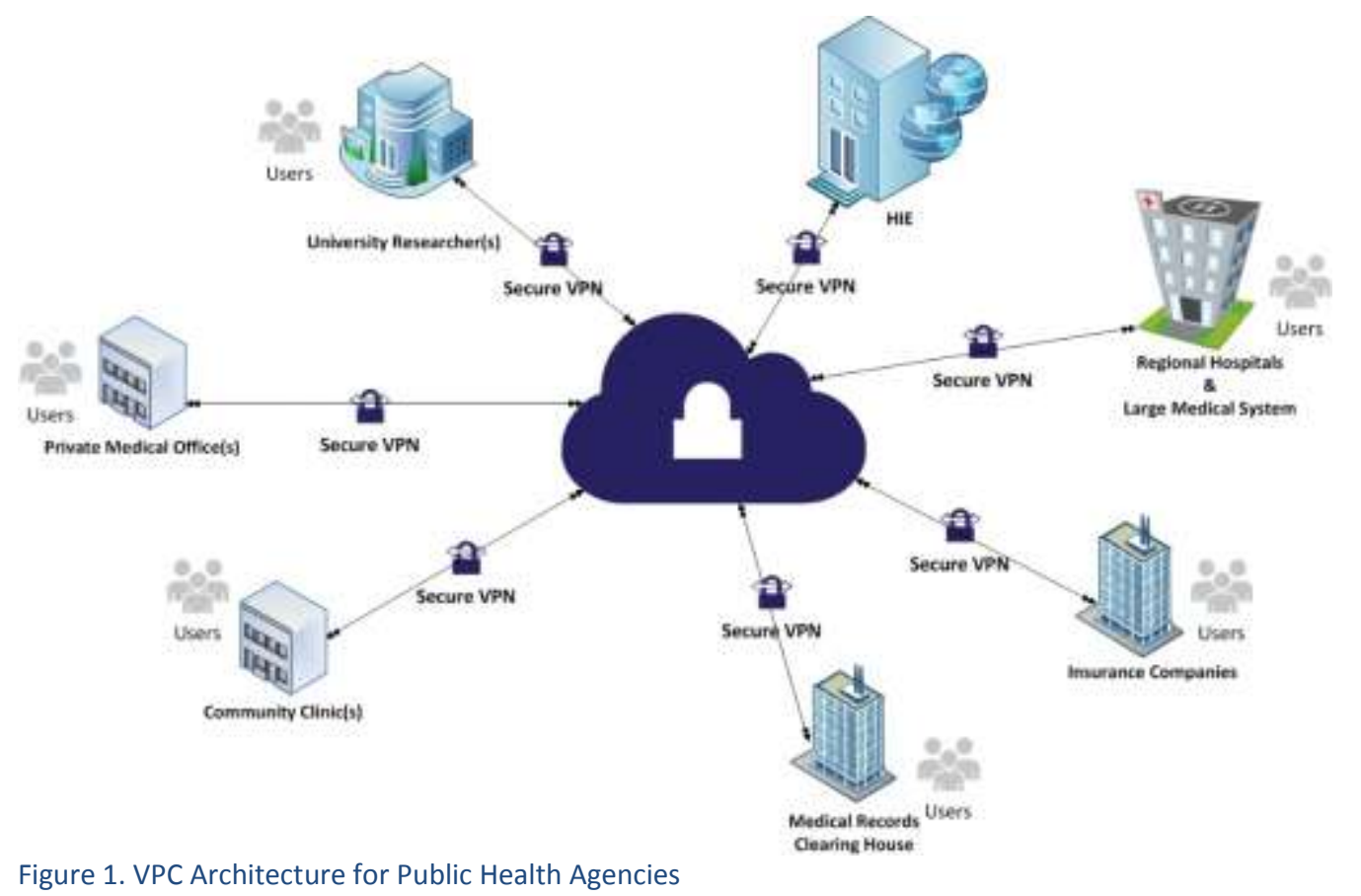


It is essentially the opposite of the most common type of cloud infrastructures, called "multitenant." In a multi-tenant architecture, multiple consumers or "tenants" share the same resources, which can lead to increased security risks and data isolation issues.

In short, a VPC has isolation and security characteristics similar to private clouds, but retains the flexibility of usage and connection common to public clouds.

\section{What are the Advantages of a VPC?}

The VPC architecture provides several significant advantages:

Security: The architecture is well suited to meet the compliance, privacy and security requirements imposed on health care organizations to safeguard individually identifiable health information (IIHI) by the Health Insurance Portability and Accountability Act of 1996 (HIPAA) as modified by the HITECH Act and its implementing regulations.

Isolation: Data within VPCs are separated from other VPCs at the network layer. This eliminates the opportunity for data or information from one cloud infrastructure mingling with those of another cloud infrastructure.

Flexibility: The VPC architecture would allow for EHR exchange among physician clinics, hospitals, electronic clearinghouses, insurance providers and promote potential researchers at universities to collaborate on the data for innovation to improve health care while reducing medical costs.

\section{Will VPC Transform PHI?}

Public health agencies are a hub for population health big data. As eligible professionals and hospitals adopt CEHRT and climb the Meaningful Use ladder, public health agencies are required to increase their technical capacities to send and receive health data securely. ${ }^{7,} 9$ Utilizing the captured data from various sources, including HIEs, to create a population health record (PopHR) for serving communities, PHI will be the trusted source of PopHR. ${ }^{14}$ With the introduction of VPC, as shown in Figure 1, public health agencies can remove information technology obstacles including, hardware and software interoperability, and network or bandwidth capacity barriers. By moving all computing resources into a VPC environment, public health agencies will have the capability to analyze the increasing data streams. Converted data stored in the VPC environment allows for the extraction of real-time knowledge. The SPHPS converts a patient centric view of health data into population centric view of health data, as shown in Figure 2.

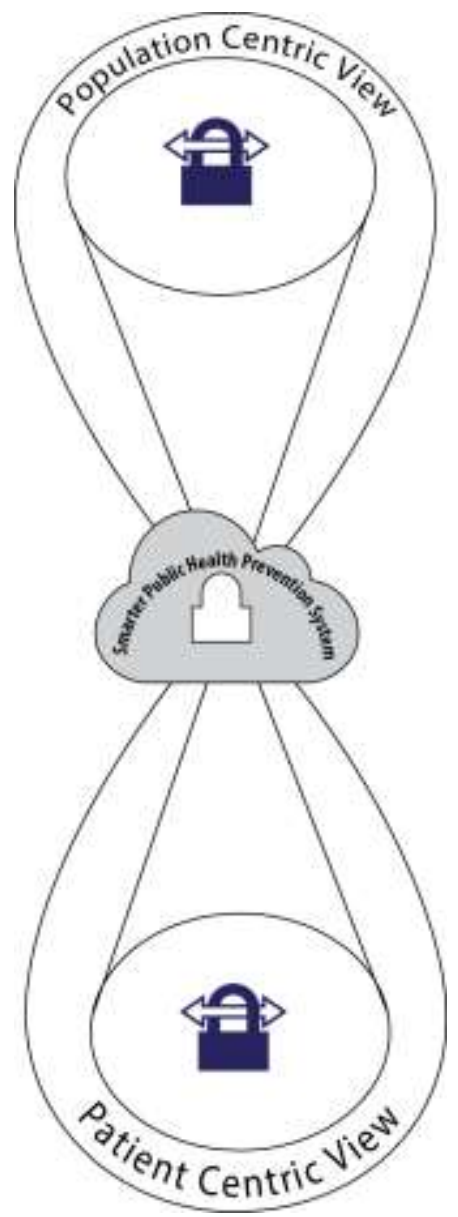

Figure 2. SPHPS Centric Views 


\section{The Case Use Scenario Perspective}

As the implementation of the SPHPS allows for the exchange of data, illustrating the SPHPS through the lens of a use case scenario can show how the data will be shared among various stakeholders. The SPHPS can estimate "population prevalence of specific conditions" by analyzing various data sources, which includes CEHRT. ${ }^{14}$ Figure 3 shows how a patient's interaction with a provider is translated into data that is then stored in the patient's EHR. Next, the patient EHR data is captured in a CEHRT; health information captured within CEHRT is securely exchanged among providers through Beacon Communities, Regional Extension Centers (REC), state health information exchanges (State HIE), and etc. SPHPS accesses the patientcentric CEHRT data and removes the IIHI information to create a PopHR. Moreover, data from various sources like public health agencies, National Center for Health Statistics (NCHS), and Data.gov, is added to create what we call big data contextual awareness within PopHR. ${ }^{14,}{ }^{15}$ Finally, the PopHR information is made accessible through a dashboard and mobile platforms. Public health professional can utilize the PopHR to create community-based interventions, and a clinicians can use this data to reduce contextual error, which are created when "a physician does not identify elements of a patient's environment or behavior, such as access to care, that must be addressed to appropriately plan care." ${ }^{, 14,15}$

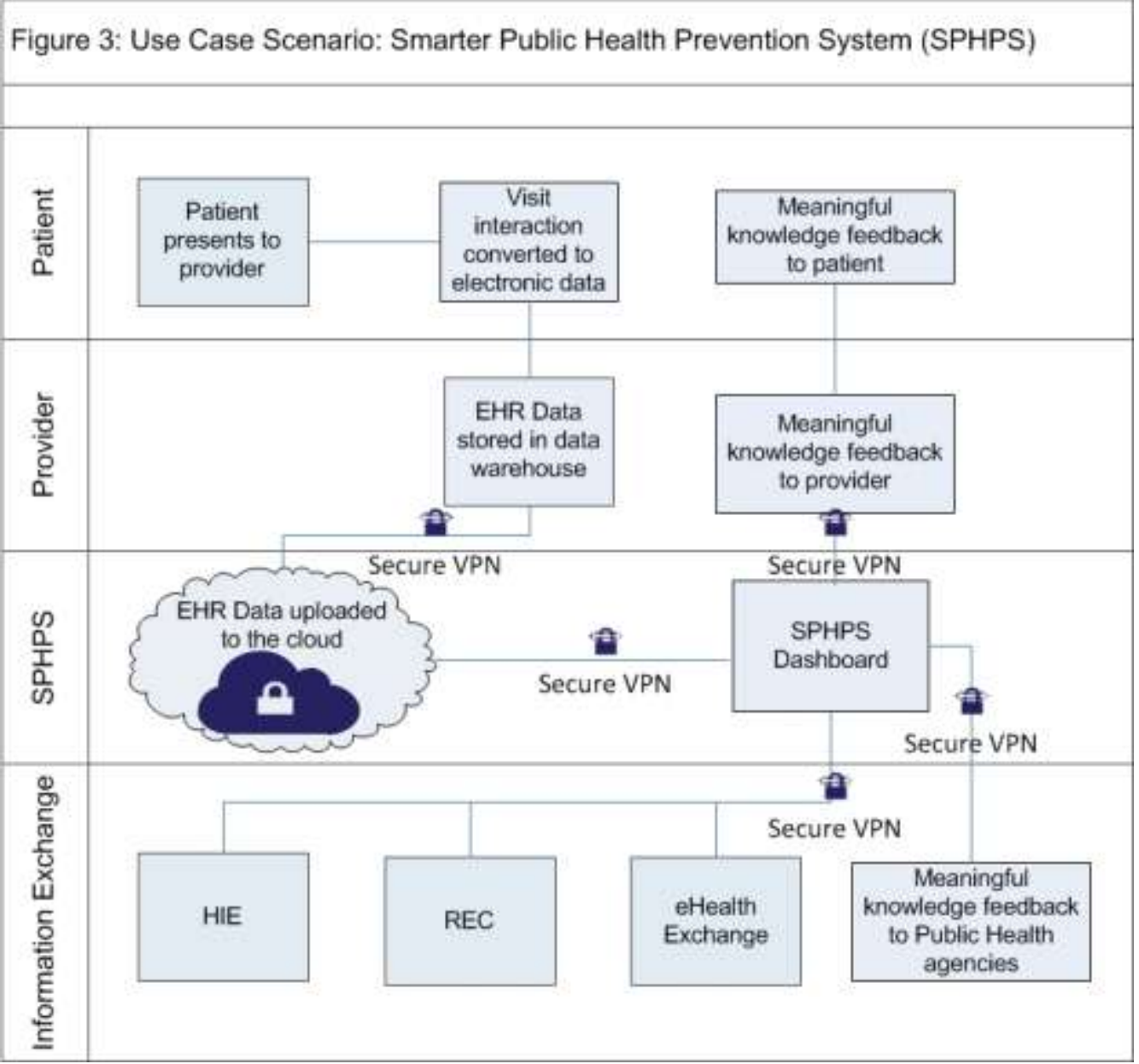

\section{Conclusion}


Cloud computing solutions can bring tremendous benefits to public health agencies and health care organizations as well as help them enhance health status or quality-of-life outcomes in atrisk populations, eliminate health disparities, and reduce overall health care costs. ${ }^{3,10,11}$ VPC offers a flexible, isolated, and secure environment for public health informaticists to gather patient centric data that can be used to reduce disparities in population health.

Cloud computing can foster sharing information between public health and health care organizations, which are stored across disparate information systems, for real-time analytics and shared decision making. It can also free up health care staff to attend to more critical tasks in an efficient, secure, and cost-effective manner.

SPHPS will securely incorporate population centric view and patient centric view to form a cloud-knowledge discovery environment. VPC will solve the HIPAA compliance issues for public health agencies and health care organizations to drive knowledge based decisions.

SPHPS can be a hub of PopHR for reducing health disparities in our communities, promotion and encouragement of healthy behaviors in our communities, prevention of epidemics and spread of disease, and primary source of secondary usage of health data in the new era of HIEs. ${ }^{3,14,16}$ Thus, the over-arching goal of SPHPS extends the current PHI knowledge in ways that will have the maximum impact on population health. ${ }^{3}$ We can achieve this goal by accelerating our knowledge discovery and preventive strategies through cloud computing. SPHPS offers the possibility of promoting healthy and safe communities. ${ }^{11}$ SPHPS can eliminate health disparities while reducing escalating health care costs and services.

\section{Acknowledgments}

The authors would like to thank John McDonald, Chief Executive Officer, CloudOne and Vishal Kapashi, Chief Architectural Officer, CloudOne.

\section{Corresponding Author}

Arash Jalali, MPH PHI

jalali@uic.edu

\section{References}

1. Friede A, Blum H, Mcdonald M. Public-health informatics - how information-age technology can strengthen public-health. Annu Rev Public Health. 1995;16:239-52.

2. Yasnoff WA, O'Carroll PW, Koo D, Linkins RW, Kilbourne EM. Public health informatics: Improving and transforming public health in the information age J Public Health Manag Pract. 2000 Nov;6(6):67-75.

3. Turnock, BJ. Public health : what it is and how it works. 5th ed. Burlington, MA: Jones \& Bartlett Learning; 2012 
4. O'Carroll P. Introduction to Public Health Informatics. O'Carroll PW, Yasnoff WA, Ward ME, Ripp LH, Martin EL, editors. Springer New York; 2003. 3 p DOI: 10.1007/0-387-22745$8 \_1$

5. Kotter JP. ACCELERATE! (cover story). Harv Bus Rev. 2012 11;90(11):43-58. Available from: http://search.ebscohost.com/login. aspx?direct=true \&db=buh\&AN=82532450\&site=ehostlive

6. Sittig DF, Singh H. A new sociotechnical model for studying health information technology in complex adaptive healthcare systems. Qual Saf Health Care. 2010 OCT;19:I68-74.

7. American Recovery and Reinvestment Act of 2009, H.R. 1, 111th Cong.

8. Burke T. The health information technology provisions in the american recovery and reinvestment act of 2009: Implications for public health policy and practice. Public Health Rep. 2010 JAN-FEB 2010;125(1)

9. Centers for Disease Control and Prevention. CDC: Meaningful Use Stage 1: Introduction. Available from: http://www.cdc.gov/ehrmeaningfuluse/introduction.html

10. National Priorities Partnership. National Priorities and Goals: Aligning Our Efforts to Transform America's Healthcare. Washington, DC: National Quality Forum; 2008.

11. National Prevention Council, National Prevention Strategy, Washington, DC: U.S. Department of Health and Human Services, Office of the Surgeon General, 2011.

12. Polgreen PM, Chen Y, Pennock DM, Nelson FD. Using internet searches for influenza surveillance. Clin Infect Dis. 2008 DEC 1;47(11):1443-8.

13. Carneiro HA, Mylonakis E. Google trends: A web-based tool for real-time surveillance of disease outbreaks. Clin Infect Dis. 2009 NOV 15;49(10):1557-64.

14. Friedman DJ, Parrish,R.Gibson,,II. The population health record: Concepts, definition, design, and implementation. J Am Med Inf Assoc. 2010 JUL;17(4):359-66.

15. Schwartz A, Weiner SJ, Harris IB, Binns-Calvey A. An educational intervention for contextualizing patient care and medical students' abilities to probe for contextual issues in simulated patients JAMA. 2010 Sep 15;304(11):1191-7.

16. Safran C, Bloomrosen M, Hammond WE, Labkoff S, Markel-Fox S, Tanc PC, Detmer DE. Toward a national framework for the secondary use of health data: An american medical informatics association white paper. J Am Med Inf Assoc. 2007 JAN-FEB;14(1):1-9. 